\title{
Nonlinear constitutive behavior of ferroelectric materials
}

\author{
LI HaiJun, LIU Feng \& WANG TzuChiang ${ }^{\dagger}$ \\ LNM, Institute of Mechanics, Chinese Academy of Sciences, Beijing 100190, China
}

The ferroelectric specimen is considered as an aggregation of many randomly oriented domains. According to this mechanism, a multi-domain mechanical model is developed in this paper. Each domain is represented by one element. The applied stress and electric field are taken to be the stress and electric field in the formula of the driving force of domain switching for each element in the specimen. It means that the macroscopic switching criterion is used for calculating the volume fraction of domain switching for each element. By using the hardening relation between the driving force of domain switching and the volume fraction of domain switching calibrated, the volume fraction of domain switching for each element is calculated. Substituting the stress and electric field and the volume fraction of domain switching into the constitutive equation of ferroelectric material, one can easily get the strain and electric displacement for each element. The macroscopic behavior of the ferroelectric specimen is then directly calculated by volume averaging. Meanwhile, the nonlinear finite element analysis for the ferroelectric specimen is carried out. In the finite element simulation, the volume fraction of domain switching for each element is calculated by using the same method mentioned above. The interaction between different elements is taken into account in the finite element simulation and the local stress and electric field for each element is obtained. The macroscopic behavior of the specimen is then calculated by volume averaging. The computation results involve the electric butterfly shaped curves of axial strain versus the axial electric field and the hysteresis loops of electric displacement versus the electric field for ferroelectric specimens under the uniaxial coupled stress and electric field loading. The present theoretical prediction agrees reasonably with the experimental results.

volume fraction of domain switching, multi-domain mechanical model, finite element method (FEM), domain switching

Received August 8, 2007; accepted January 19, 2008 doi: $10.1007 / \mathrm{s} 11433-008-0140-4$

${ }^{\dagger}$ Corresponding author (email: tcwang@imech.ac.cn)

Supported by the National Natural Science Foundation of China (Grant No. 10572138) 


\section{Introduction}

Ferroelectric materials are used in many electric components such as sensors, actuators and transducers owing to their coupled electromechanical characteristics. As the core device of smart structures they are used in aerospace, precise instruments, automatic control and micro electromechanical system and so on. When the applied electric field approaches or exceeds the coercive electric field, the classical linear piezoelectric theory is not suitable. There have been extensive research efforts in the field of constitutive modeling and numerical simulation of the nonlinear behavior of ferroelectric materials.

Many theories were proposed to explain the nonlinear behavior of the ferroelectric ceramics. Different types of constitutive models for the ferroelectric ceramics can be classified into two groups. One group is based on the micro-electro-mechanical behavior of grains and the second on the macro phenomenological model.

Domain switching is the important source of the nonlinear response of ferroelectric ceramics. The micro-electro-mechanical model is based on the microscopic behavior of the domains. As a result, the model has the ability to capture much of the underlying physical behavior. In the model, a number of randomly oriented domains are subjected to the electrical and/or mechanical loading. At each loading increment, some switching criterion is applied to each domain, and if the switching criterion can be reached or exceeded, the domain switching takes place. After each increment of the applied field and updating of the polarization, the volume average and the polarization are calculated ${ }^{[1-10]}$. Huber et al. ${ }^{[11]}$ developed the ferroelectric constitutive model based on the domain wall motion that is similar to the crystal slip. They thought that there were several variants in a tetragonal crystal and the volume fraction of each variant was $c_{I}$. They used a self-consistent analysis to estimate the macroscopic response of the tetragonal crystals under a variety of loading paths. Huber and Fleck ${ }^{[12]}$ improved this model. Chen et al. ${ }^{[13]}$ introduced the volume fraction of various kinds of domains as internal variables that describ the pattern of the internal rearrangement resulting from domain switching and studied the nonlinear behavior of polycrystalline ferroelectrics. Lu et al. ${ }^{[14,15]}$ developed a domain-switching model with the consideration of electromechanical interactions in a self-consistent manner and the use of an ODF. Micro-electro-mechanical model reflects the physical essence of the nonlinear behavior of the ferroelectric material. But in order to make the calculation accurate, those methods require thousands of crystallite grains. Hence the finite element simulations cost too much computation time and are not efficient.

In order to make the complex ferroelectric nonlinear constitutive behavior available for structural mechanical analysis, the phenomenological models with fewer internal variables have been developed. Chen and his cooperators ${ }^{[16-19]}$ proposed the phenomenological model to describe the electromechanical coupling characteristics of the ferroelectric material. They used the remnant polarization and the remnant strain as the internal variables and they only considered the uniaxial loading. Another attempt to study the nonlinear constitutive behavior of the ferroelectric materials was made by Bassiouny and Maugin ${ }^{[20,21]}$. They presented a thermodynamically phenomenological theory to describe the constitutive behavior of the ferroelectric ceramics. The theory considers both the plastic and the electric-hysteresis effects in the form of "plasticity". A simple macroscopic constitutive model with remnant polarization and remnant strain as internal variables was developed by Kamlah and his coworkers ${ }^{[22-25]}$. The model can be used to study the fully coupled elec- 
tromechanical boundary value problem. A phenomenological theory was also presented by Cocks and McMeeking ${ }^{[26-28]}$. The model is established in the framework of traditional incremental plasticity. Landis ${ }^{[29]}$ introduced nine internal variables corresponding to the components of the remnant strain and the remnant polarization to match all of the types of behaviors of the ferroelectric ceramics in the uniaxial loading. A simplified formulation with fewer internal variables was derived by McMeeking and Landis ${ }^{[28]}$. In the model, only three internal variables are employed by linking the remnant strain to the remnant polarization. Due to this simplicity, the switching criterion can be expressed by a modified electric field variable and the kinematic hardening potential can be given as a function of the remnant polarization. A detailed review on recent advances in the nonlinear model in ferroelectric materials was given by Kamlah ${ }^{[30]}$ and Landis ${ }^{[31]}$. So far all the phenomenological theories predicted well for the uniaxial mechanical loading and the uniaxial electric loading. But there are some deviations between the theoretical prediction and experimental results under the uniaxial electromechanical coupling loading.

As for the nonlinear finite element analysis in ferroelectric material, Gong and Suo ${ }^{[32]}$, Hom and Shankar ${ }^{[33]}$ simulated the electrostriction behavior of the multilayer actuators. Based on the switching criterion, the interaction among grains has been implemented in the finite element method for simulating the fully coupled constitutive behavior by Hwang et al ${ }^{[6]}$, Lu et al. ${ }^{[4]}$, Hwang and Waser ${ }^{[34]}$. Liu ${ }^{[35]}$ analyzed the plane problem using the fully coupled nonlinear finite element method. Li and Fang ${ }^{[36]}$ carried out the three-dimensional finite element simulations on ferroelectric materials. The calculation was difficult because of the large elastic strain mismatch during the domain switching.

Cao and Evans $\frac{[37]}{3}$ carried out a preliminary experimental study for the nonlinear behavior of the ceramics PZT and PLZT under both electric loadings and mechanical loadings. An experimental measurement on the nonlinear behavior of PLZT was presented by Hwang et al. ${ }^{[2]}$ under the uniaxial compressive stress and the electric field loading. A systematic experimental study on the electro-mechanical behavior of the ceramics PLZT under the uniaxial electro-mechanical coupled loading was reported by $\operatorname{Lynch}^{[38]}$. A similar experimental study was given by Fang and $\mathrm{Li}^{[39]}$. Huber and Fleck ${ }^{[11]}$ studied the nonlinear constitutive response on PZT under the multi-axial electric loading.

\section{Constitutive law}

The physical experiments given by Miller and Weinreich ${ }^{[40]}$ and Hayashi ${ }^{[41]}$ showed that domain switching in the ferroelectric material was a complicated process. It is composed of four stages: nucleation, longitudinal growth, transverse expansion and merging of the new domains ${ }^{[42]}$. Hence the domain switching in each single domain is an evolution process of the domain wall motion.

Following Hwang et al. ${ }^{[6]}$, McMeeking, Landis ${ }^{[28]}$ and Kamlah ${ }^{[30]}$, we assume the ferroelectric material to be isotropic elastic and isotropic dielectric for simplicity. But the piezoelectric coefficient $d_{k i j}$ will be changed due to domain switching. First, considering the domain switches only in one direction. Supposing that the material element is a single domain zone before switching, the spontaneous polarization, the spontaneous polarization strain and the piezoelectric coefficient are $D_{i}^{s(0)}, \quad \gamma_{i j}^{s(0)}$ and $d_{i j k}^{(0)}$, respectively. The corresponding quantities will be $D_{i}^{s(I)}, \gamma_{i j}^{s(I)}$ and 
$d_{i j k}^{(I)}$ after the whole domain in the material element has switched. If the volume fraction of the switched domain in the material element is $\rho_{I}$, the spontaneous polarization, the spontaneous strain and the piezoelectric coefficient of the material element will be

$$
\begin{aligned}
& D_{i}^{s}=\left(1-\rho_{I}\right) D_{i}^{s(0)}+\rho_{I} D_{i}^{s(I)}=D_{i}^{s(0)}+\rho_{I} \Delta D_{i}^{s(I)}, \\
& \gamma_{i j}^{s}=\left(1-\rho_{I}\right) \gamma_{i j}^{s(0)}+\rho_{I} \gamma_{i j}^{s(I)}=\gamma_{i j}^{s(0)}+\rho_{I} \Delta \gamma_{i j}^{s(I)}, \\
& d_{i k l}=\left(1-\rho_{I}\right) d_{i k l}^{(0)}+\rho_{I} d_{i k l}^{(I)}=d_{i k l}^{(0)}+\rho_{I} \Delta d_{i k l}^{(I)},
\end{aligned}
$$

where symbol $\Delta$ is the change of the related physical quantity before switching and after the whole domain of the material element having switched.

In fact, the domain may switch both in the $90^{\circ}$ and $180^{\circ}$ directions. And the domain may switch in several potential orientations during $90^{\circ}$ switching. Suppose the volume fraction of $180^{\circ}$ switching is $\rho_{1}$. If there are $N-190^{\circ}$ switching, the volume fraction of $90^{\circ}$ switching can be expressed as $\rho_{I}(I \in 2,3, \ldots, N)$.

Similar to eq. (1), the spontaneous electric displacement, the spontaneous strain and the piezoelectric coefficient of the material element can be written as follows:

$$
\begin{gathered}
D_{i}^{s}=D_{i}^{s(0)}+\sum_{I=1}^{N} \rho_{I} \Delta D_{i}^{s(I)}, \\
\gamma_{i j}^{s}=\gamma_{i j}^{s(0)}+\sum_{I=1}^{N} \rho_{I} \Delta \gamma_{i j}^{s(I)}, \\
d_{i k l}=d_{i k l}^{(0)}+\sum_{I=1}^{N} \rho_{I} \Delta d_{i k l}^{(I)},
\end{gathered}
$$

where $I=1$ corresponds to $180^{\circ}$ switching, and $I \in 2,3, \ldots, N$ are corresponding to $90^{\circ}$ switching in the $I$ th orientation.

The constitutive law of the ferroelectric material can be described as follows:

$$
\left\{\begin{array}{l}
\gamma_{i j}-\gamma_{i j}^{s(0)}-\sum_{I=1}^{N} \rho_{I} \Delta \gamma_{i j}^{s(I)}=S_{i j k l} \sigma_{k l}+d_{k i j} E_{k}=S_{i j k l} \sigma_{k l}+\left(d_{k i j}^{(0)}+\sum_{I=1}^{N} \rho_{I} \Delta d_{k i j}^{(I)}\right) E_{k}, \\
D_{i}-D_{i}^{s(0)}-\sum_{I=1}^{N} \rho_{I} \Delta D_{i}^{s(I)}=d_{i k l} \sigma_{k l}+\varepsilon_{i k} E_{k}=\left(d_{i k l}^{(0)}+\sum_{I=1}^{N} \rho_{I} \Delta d_{i k l}^{(I)}\right) \sigma_{k l}+\varepsilon_{i k} E_{k},
\end{array}\right.
$$

where $S_{i j k l}$ and $\varepsilon_{i k}$ are the elastic compliance and dielectric permittivity tensor measured at constant electric field and constant stress, respectively. Tensor $d_{i k l}$ is the piezoelectric coefficient at constant stress. In general, the displacement $u_{i}$ and the electric potential $\phi$ are used as the basic unknown quantities in the finite element method. Hence the second type constitutive law is needed, in which the basic unknown quantities are the strain $\gamma_{i j}$ and the electric field $E_{i}$. We have

$$
\begin{aligned}
& \sigma_{i j}=C_{i j m n}\left(\gamma_{m n}-\gamma_{m n}^{s}\right)-C_{i j m n} d_{k m n} E_{k}, \\
& D_{i}=D_{i}^{s}+C_{m n k l} d_{i k l}\left(\gamma_{m n}-\gamma_{m n}^{s}\right)+\left(\varepsilon_{i k}-d_{k m n} C_{m n l j} d_{i l j}\right) E_{k},
\end{aligned}
$$

where $C_{i j m n}$ is the elastic modulus tensor measured at constant electric field. 


\section{Mechanical model}

As shown in the constitutive experiments, linear models of many piezoelectric materials are valid only within a limited range of loading. If a sufficiently large electric field is applied to the ferroelectric materials, the domain switching takes place and changes the direction of polarization to that most closely aligned with the electric field. The nonlinear response occurs mainly due to this domain switching. The domain can switch by $90^{\circ}$ or $180^{\circ}$. The $90^{\circ}$ switching is accompanied by a change of the spontaneous strain and the spontaneous polarization, but $180^{\circ}$ switching only changes the spontaneous polarization.

\subsection{Single domain mechanical model}

Single domain mechanical model was presented in ref. [1]. The ferroelectric ceramic is poled by an electric field in excess of the coercive field, thus aligning the polarization of each domain as closely as possible with the applied field. In ref. [1], the specimen of the poled ferroelectric ceramic was considered as a transversely isotropic single domain material, and the hardening relation between the driving force of domain switching and the volume fraction of domain switching can be calibrated based on the partial experiment results for the poled ferroelectric ceramic specimen.

The switching criterion proposed by Hwang et al. ${ }^{[2]}$ can be expressed as

$$
E_{i} \Delta D_{i}^{s(I)}+\sigma_{i j} \Delta \gamma_{i j}^{s(I)}=W_{c r}^{(I)} .
$$

The initial energy barrier against $90^{\circ}$ switching is assumed to be $\frac{3}{2} \sigma_{c} \gamma^{s}$, and the initial energy barrier against $180^{\circ}$ switching is $2 E_{c} P_{s} . \sigma_{c}$ is the coercive stress, and $\gamma^{s}$ is the spontaneous strain. The energy barrier will be strengthened with the increase of the volume fraction of domain switching. The hardening relation can be dimensionless by the initial energy barrier:

$$
\begin{gathered}
f_{1}\left(\rho_{1}\right)=\frac{W_{c r}^{(1)}}{2 E_{c} P_{s}}, \\
f_{2}\left(\rho_{2}\right)=\frac{W_{c r}^{(2)}}{1.5 \sigma_{c} \gamma^{s}} .
\end{gathered}
$$

The hardening relation between the driving force of domain switching and the volume fraction of domain switching has been given in ref. [1]:

(1) Purely electric loadings. The hardening function of $90^{\circ}$ domain switching is given by

$$
f_{2}\left(\rho_{2}\right)=1+1.289 \times \frac{\left(1-\mathrm{e}^{-2.588 \rho_{2}}\right)}{\left(1-\mathrm{e}^{-2.588}\right)}+3.711 \times \frac{\left(1-\mathrm{e}^{-15.47 \rho_{2}}\right)}{\left(1-\mathrm{e}^{-15.47}\right)} .
$$

(2) Purely mechanical loadings and electromechanical coupling loadings. The hardening relation of $90^{\circ}$ domain switching is quite similar in these two cases. The hardening function is expressed as

$$
f_{2}\left(\rho_{2}\right)=\frac{7.340 \rho_{2}}{0.2790+\rho_{2}}+\frac{1.835 \rho_{2}}{0.9799-\rho_{2}}-6.256 \rho_{2}+1
$$

(3) Plastic strain. The irreversible strain is generated because of compacting the internal micro void in ferroelectric ceramics:

$$
\gamma_{33}^{p}=0.01477\left(\mathrm{e}^{-0.5856 \sigma_{33} / \sigma_{c}}-1\right), \quad-\sigma_{33} / \sigma_{c}<3.57,
$$




$$
\gamma_{33}^{p}=-0.125 \%, \quad-\sigma_{33} / \sigma_{c} \geqslant 3.57 .
$$

(4) Purely electric loadings and electromechanical coupling loadings. The hardening function of $180^{\circ}$ domain switching is

$$
f_{1}\left(\rho_{1}\right)=E_{c}^{\sigma} / E_{c}+B \cdot \rho_{1},
$$

where

$$
B=0.0242-0.3607 \sigma_{33} / \sigma_{c} .
$$

(5) Unloading. When the electric unloading occurs after the negative electric load arrives the maximum value, the $90^{\circ}$ and $180^{\circ}$ domain switchings cannot occur under purely mechanical loadings and purely electric loadings since the driving force decreases. So the constitutive behavior is linear. As for the combined electromechanical loadings, the $180^{\circ}$ domain switching cannot occur. But the $90^{\circ}$ domain switching will take place when the driving force of domain switching is greater than the value of hardening function. Then the volume fraction of $90^{\circ}$ switching is calculated according to eq. (9).

\subsection{Multi-domain mechanical model}

The present calculations are based on the multi-domain mechanical model. The poled ferroelectric ceramics is considered as an aggregation of many randomly domains. In the case of plane strain, only clockwise $90^{\circ}$ switching, counter clockwise $90^{\circ}$ switching and $180^{\circ}$ switching are considered in this paper. The detailed description of the calculation method is given in the next section.

\section{Finite element formulation}

Typical piezoelectric finite element models by using displacements and the electric potential as nodal degrees of freedom are employed in this paper. The finite element software is programmed according to the underlying equations.

Generalized geometric equations are

$$
\begin{gathered}
\gamma_{i j}=\frac{1}{2}\left(u_{i, j}+u_{j, i}\right), \\
E_{i}=-\phi_{, i} .
\end{gathered}
$$

The second type of the constitutive law of the ferroelectric materials is used in the finite element method:

$$
\begin{gathered}
\sigma_{i j}=C_{i j m n}\left(\gamma_{m n}-\gamma_{m n}^{s}\right)-e_{k i j} E_{k}, \\
D_{i}=D_{i}^{s}+e_{i m n}\left(\gamma_{m n}-\gamma_{m n}^{s}\right)+\varepsilon_{i k}^{\gamma} E_{k},
\end{gathered}
$$

where

$$
\begin{gathered}
C_{i j k l}=\frac{E}{1+v}\left(\frac{1}{2}\left(\delta_{i k} \delta_{j l}+\delta_{i l} \delta_{j k}\right)+\frac{v}{1-2 v} \delta_{i j} \delta_{k l}\right), \\
d_{k i j}^{(I)}=d_{33} n_{k}^{(I)} n_{i}^{(I)} n_{j}^{(I)}+d_{31} n_{k}^{(I)} \alpha_{i j}+\frac{1}{2} d_{15}\left(n_{i}^{(I)} \alpha_{i k}+n_{j}^{(I)} \alpha_{i k}\right), \\
\varepsilon_{i j}=\varepsilon_{0} \delta_{i j}, \\
e_{k i j}=C_{i j m n} d_{k m n}, \\
\varepsilon_{i k}^{\gamma}=\varepsilon_{i k}-d_{k m n} e_{i m n},
\end{gathered}
$$




$$
\alpha_{i j}=\delta_{i j}-n_{i}^{(I)} n_{j}^{(I)},
$$

where $n_{i}^{(0)}$ is the component of the unit vector in the initial polarization direction; $n_{i}^{(I)}$ is the component of the unit vector in the switched polarization direction; $d_{33}, d_{31}$ and $d_{15}$ are the longitudinal, transverse and shear piezoelectric constants of a single domain of the ferroelectric materials; $\varepsilon_{0}$ is the dielectric permittivity.

Suppose that the displacement $u_{i}$ and the electric potential $\phi$ satisfy the boundary conditions, the field equation and the boundary conditions are equivalent to the following variational equation:

$$
\int_{V}\left(\sigma_{i j, i} \delta u_{j}+D_{i, i} \delta \phi\right) \mathrm{d} V-\int_{S_{\sigma}}\left(\sigma_{i j} n_{i}-\bar{t}_{j}\right) \delta u_{j} \mathrm{~d} S-\int_{S_{\omega}}\left(D_{i} n_{i}+\bar{\omega}\right) \delta \phi \mathrm{d} S=0
$$

It must be emphasized that eq. (23) is only suitable for the fixed volume fraction $\rho_{I}$ of domain switching. It means that the volume fraction $\rho_{I}$ of domain switching is not subjected to the variation in eq. (23). The first term of the left part in eq. (23) can be written as

$$
\int_{V}\left(\sigma_{i j, i} \delta u_{j}+D_{i, i} \delta \phi\right) \mathrm{d} V=\int_{V}\left(-\sigma_{i j} \delta u_{j, i}-D_{i} \delta \phi_{, i}\right) \mathrm{d} V+\int_{S} \sigma_{i j} n_{i} \delta u_{j} \mathrm{~d} S+\int_{S} D_{i} n_{i} \delta \phi \mathrm{d} S .
$$

Furthermore, we have

$$
\begin{aligned}
& \int_{V}\left(-\sigma_{i j} \delta u_{j, i}-D_{i} \delta \phi_{, i}\right) \mathrm{d} V \\
& =\int_{V}\left(-\sigma_{i j} \delta \gamma_{i j}+D_{i} \delta E_{i}\right) \mathrm{d} V \\
& =\int_{V}\left\{-\left[C_{i j m n}\left(\gamma_{m n}-\gamma_{m n}^{s}\right)-e_{k i j} E_{k}\right] \delta \gamma_{i j}+\left[e_{i m n}\left(\gamma_{m n}-\gamma_{m n}^{s}\right)+\varepsilon_{i k}^{\gamma} E_{k}+D_{i}^{s}\right] \delta E_{i}\right\} \mathrm{d} V \\
& =\delta\left[\int_{V}\left(-\frac{1}{2} C_{i j k l} \gamma_{k l} \gamma_{i j}+e_{k i j} E_{k} \gamma_{i j}+\frac{1}{2} \varepsilon_{i k}^{\gamma} E_{i} E_{k}\right) \mathrm{d} V\right]+\int_{V}\left[\left(D_{i}^{s}-e_{i j k} \gamma_{j k}^{s}\right) \delta E_{i}+C_{i j k l} \gamma_{k l}^{s} \delta \gamma_{i j}\right] \mathrm{d} V .
\end{aligned}
$$

Substituting eq. (25) into eq. (24), then into eq. (23), we can get

$$
\begin{aligned}
& \delta\left[\int_{V}\left(\frac{1}{2} C_{i j k l} \gamma_{k l} \gamma_{i j}-e_{k i j} E_{k} \gamma_{i j}-\frac{1}{2} \varepsilon_{i j}^{\gamma} E_{i} E_{j}\right) \mathrm{d} V\right]-\int_{V}\left[C_{i j k l} \gamma_{k l}^{s} \delta \gamma_{i j}-\left(e_{i j k} \gamma_{j k}^{s}-D_{i}^{s}\right) \delta E_{i}\right] \mathrm{d} V \\
& -\int_{S_{\sigma}} \bar{t}_{j} \delta u_{j} \mathrm{~d} S+\int_{S_{\omega}} \bar{\omega} \delta \phi \mathrm{d} S=0 .
\end{aligned}
$$

Hence the total potential energy function of the ferroelectric body can be expressed as

$$
\begin{aligned}
\Pi= & \int_{V}\left(\frac{1}{2} C_{i j k l} \gamma_{k l} \gamma_{i j}-e_{k i j} E_{k} \gamma_{i j}-\frac{1}{2} \varepsilon_{i j}^{r} E_{i} E_{j}\right) \mathrm{d} V-\int_{V}\left[C_{i j k l} \gamma_{k l}^{s} \gamma_{i j}-\left(e_{i j k} \gamma_{j k}^{s}-D_{i}^{s}\right) E_{i}\right] \mathrm{d} V \\
& -\int_{S_{\sigma}} \bar{t}_{j} u_{j} \mathrm{~d} S+\int_{S_{\omega}} \bar{\omega} \phi \mathrm{d} S .
\end{aligned}
$$

The variation principle is

$$
\delta \Pi=0 .
$$

Linear triangle elements are used in the 2D problem. In the plane strain problem of $x-z$ plane, the generalized displacement is

$$
\boldsymbol{u}=\left[\begin{array}{lll}
u_{x} & u_{z} & \phi
\end{array}\right]^{\mathrm{T}} .
$$

The generalized strain is

$$
\boldsymbol{\Gamma}=\left[\begin{array}{lllll}
\gamma_{x} & \gamma_{z} & 2 \gamma_{x z} & -E_{x} & -E_{z}
\end{array}\right]^{\mathrm{T}}=\left[\begin{array}{lllll}
\gamma_{11} & \gamma_{33} & 2 \gamma_{13} & -E_{1} & -E_{3}
\end{array}\right]^{\mathrm{T}} .
$$

The generalized stress is 


$$
\Sigma=\left[\begin{array}{lllll}
\sigma_{x} & \sigma_{z} & \sigma_{x z} & D_{x} & D_{z}
\end{array}\right]^{\mathrm{T}}=\left[\begin{array}{lllll}
\sigma_{11} & \sigma_{33} & \sigma_{13} & D_{1} & D_{3}
\end{array}\right]^{\mathrm{T}} .
$$

The generalized nodal displacement array is

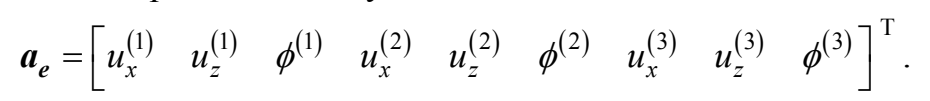

The generalized displacement in the elements can be interpolated as

$$
\begin{gathered}
\boldsymbol{u}=\boldsymbol{N} \boldsymbol{a}_{\boldsymbol{e}}, \\
\boldsymbol{N}=\left[\begin{array}{cccccccccc}
a_{i}+b_{i} x+c_{i} z & 0 & 0 & a_{j}+b_{j} x+c_{j} z & 0 & 0 & a_{m}+b_{m} x+c_{m} z & 0 & 0 \\
0 & a_{i}+b_{i} x+c_{i} z & 0 & 0 & a_{j}+b_{j} x+c_{j} z & 0 & 0 & a_{m}+b_{m} x+c_{m} z & 0 \\
0 & 0 & a_{i}+b_{i} x+c_{i} z & 0 & 0 & a_{j}+b_{j} x+c_{j} z & 0 & 0 & a_{m}+b_{m} x+c_{m} z
\end{array}\right],
\end{gathered}
$$

where $a_{i}, b_{i}, c_{i}, a_{j}, b_{j}, c_{j}, a_{m}, b_{m}, c_{m}$ is constant. The strain can be expressed as follows:

$$
\Gamma=L u=L N a_{e}=B a_{e},
$$

where

$$
\begin{aligned}
\boldsymbol{L} & =\left[\begin{array}{lll}
\frac{\partial}{\partial x} & 0 & 0 \\
0 & \frac{\partial}{\partial z} & 0 \\
\frac{\partial}{\partial z} & \frac{\partial}{\partial x} & 0 \\
0 & 0 & \frac{\partial}{\partial x} \\
0 & 0 & \frac{\partial}{\partial z}
\end{array}\right], \\
\boldsymbol{B} & =\left[\begin{array}{ccccccccc}
b_{i} & 0 & 0 & b_{j} & 0 & 0 & b_{m} & 0 & 0 \\
0 & c_{i} & 0 & 0 & c_{j} & 0 & 0 & c_{m} & 0 \\
c_{i} & b_{i} & 0 & c_{j} & b_{j} & 0 & c_{m} & b_{m} & 0 \\
0 & 0 & b_{i} & 0 & 0 & b_{j} & 0 & 0 & b_{m} \\
0 & 0 & c_{i} & 0 & 0 & c_{j} & 0 & 0 & c_{m}
\end{array}\right] .
\end{aligned}
$$

The relationship between the generalized stress and the generalized strain is

$$
\Sigma=C\left(\Gamma-\Gamma^{s}\right)+D^{s},
$$

where

$$
\begin{aligned}
\boldsymbol{C}= & {\left[\begin{array}{ccccc}
C_{1111} & C_{1133} & 0 & e_{111} & e_{311} \\
C_{3311} & C_{3333} & 0 & e_{133} & e_{333} \\
0 & 0 & C_{1313} & e_{131} & e_{331} \\
e_{111} & e_{133} & e_{113} & -\varepsilon_{11}^{\gamma} & -\varepsilon_{13}^{\gamma} \\
e_{311} & e_{333} & e_{313} & -\varepsilon_{31}^{\gamma} & -\varepsilon_{33}^{\gamma}
\end{array}\right], } \\
& \boldsymbol{\Gamma}^{s}=\left[\begin{array}{lllll}
\gamma_{11}^{s} & \gamma_{33}^{s} & 2 \gamma_{13}^{s} & 0 & 0
\end{array}\right]^{\mathrm{T}},
\end{aligned}
$$




$$
\boldsymbol{D}^{s}=\left[\begin{array}{lllll}
0 & 0 & 0 & D_{1}^{s} & D_{3}^{s}
\end{array}\right]^{\mathrm{T}} .
$$

The total potential energy $\Pi$ in eq. (27) can be expressed by the generalized nodal displacement $\boldsymbol{a}_{\boldsymbol{e}}$. Suppose the thickness of the body is unit. Hence we have

$$
\begin{aligned}
& \Pi=\sum\left(\boldsymbol{a}_{e}^{\boldsymbol{T}} \int_{\Omega_{e}} \frac{1}{2} \boldsymbol{B}^{\boldsymbol{T}} \boldsymbol{C B} \boldsymbol{a}_{\boldsymbol{e}} \mathrm{d} x \mathrm{~d} y\right) \\
&-\sum\left(\boldsymbol{a}_{\boldsymbol{e}}^{\boldsymbol{T}} \int_{\Omega_{e}} \boldsymbol{B}^{\boldsymbol{T}}\left(\boldsymbol{C} \boldsymbol{\Gamma}^{\boldsymbol{s}}-\boldsymbol{D}^{\boldsymbol{s}}\right) \mathrm{d} x \mathrm{~d} y\right)-\sum\left(\boldsymbol{a}_{\boldsymbol{e}}^{\boldsymbol{T}} \int_{S_{\sigma}} \boldsymbol{N}^{\boldsymbol{T}} \overline{\boldsymbol{T}} \mathrm{d} S\right)+\sum\left(\boldsymbol{a}_{\boldsymbol{e}}^{\boldsymbol{T}} \int_{S_{\omega}} \boldsymbol{N}^{\boldsymbol{T}} \bar{\omega} \mathrm{d} S\right), \\
& \overline{\boldsymbol{T}}=\left[\begin{array}{lll}
\overline{1} & \overline{t_{3}} & 0
\end{array}\right]^{\mathrm{T}}, \\
& \overline{\boldsymbol{\omega}}=\left[\begin{array}{lll}
0 & 0 & \bar{\omega}
\end{array}\right]^{\mathrm{T}} .
\end{aligned}
$$

The element nodal displacement $\boldsymbol{a}_{\boldsymbol{e}}$ can be expressed by the total nodal displacement $\boldsymbol{a}$, so that

$$
\boldsymbol{a}_{\boldsymbol{e}}=\boldsymbol{G a},
$$

where $\boldsymbol{G}$ is a transformation matrix related to the local and global displacement. Then we have

$$
\begin{aligned}
\Pi= & \sum \boldsymbol{a}^{\boldsymbol{T}} \boldsymbol{G}^{\boldsymbol{T}} \int_{\Omega_{e}} \frac{1}{2} \boldsymbol{B}^{\boldsymbol{T}} \boldsymbol{C B} \boldsymbol{G} \boldsymbol{a} \mathrm{d} x \mathrm{~d} y \\
& -\sum \boldsymbol{a}^{\boldsymbol{T}} \boldsymbol{G}^{\boldsymbol{T}} \int_{\Omega_{e}} \boldsymbol{B}^{\boldsymbol{T}}\left(\boldsymbol{C} \boldsymbol{\Gamma}^{\boldsymbol{s}}-\boldsymbol{D}^{\boldsymbol{s}}\right) \mathrm{d} x \mathrm{~d} y-\sum \boldsymbol{a}^{\boldsymbol{T}} \boldsymbol{G}^{\boldsymbol{T}} \int_{S_{\sigma}} \boldsymbol{N}^{\boldsymbol{T}} \overline{\boldsymbol{T}} \mathrm{d} S+\sum \boldsymbol{a}^{\boldsymbol{T}} \boldsymbol{G}^{\boldsymbol{T}} \int_{S_{\omega}} \boldsymbol{N}^{\boldsymbol{T}} \overline{\boldsymbol{\omega}} \mathrm{d} S .
\end{aligned}
$$

Eq. (42) can be written as

$$
\Pi=\frac{1}{2} a^{T} K a-a^{T} P
$$

where the global stiffness matrix is

$$
K=\sum G^{T} K^{e} G
$$

$\boldsymbol{K}_{\boldsymbol{e}}$ is the element stiffness matrix,

$$
\boldsymbol{K}_{e}=\int_{\Omega_{e}} \boldsymbol{B}^{T} \boldsymbol{C B} \mathrm{d} x \mathrm{~d} y .
$$

$\boldsymbol{P}$ is the global external force,

$$
P=\sum G^{T} P_{\Omega}^{e}+\sum G^{T} P_{T}^{e}-\sum G^{T} P_{\omega}^{e} .
$$

$\boldsymbol{P}_{\Omega}^{e}$ represents the nodal load due to the spontaneous strain and spontaneous polarization,

$$
\boldsymbol{P}_{\Omega}^{e}=\int_{\Omega_{e}} \boldsymbol{B}^{T}\left(\boldsymbol{C} \boldsymbol{\Gamma}^{s}-\boldsymbol{D}^{s}\right) \mathrm{d} x \mathrm{~d} y .
$$

$\boldsymbol{P}_{T}^{e}$ is the nodal force due to boundary traction,

$$
\boldsymbol{P}_{T}^{e}=\int_{S_{\sigma}} N^{T} \bar{T} \mathrm{~d} S
$$

$\boldsymbol{P}_{\boldsymbol{\omega}}^{e}$ is the nodal load due to free electric charge density at the surface,

$$
\boldsymbol{P}_{\omega}^{\boldsymbol{e}}=\int_{S_{\sigma}} N^{T} \bar{\omega} \mathrm{d} S .
$$

According to the variational principle, when the functional $\Pi$ gets stationary, the first variation of the functional $\Pi$ should be zero, $\delta \Pi=0$. That is to say 


$$
\frac{\partial \Pi}{\partial \boldsymbol{a}}=0
$$

Hence we get the finite element equation:

$$
\boldsymbol{K a}=\boldsymbol{P} .
$$

We should emphasize that the variation principle (51) is only suitable for the prescribed volume fraction $\rho_{I}$ of domain switching. It means that we only take into account the variations of the displacement and the electric potential in eq. (51).

\section{The calculated model}

\subsection{Boundary conditions}

In Lynch's ${ }^{[38]}$ experimental work, a sample of $10 \mathrm{~mm}$ cube was used to obtain the experimental data. Hence strictly speaking, 3D finite element simulation should be adopted. But the 3D finite element simulation costs too much computation time and the efficiency of the 3D finite element simulation is quite low due to the complex distribution of the polarization direction. For simplicity, a 2D finite element model is proposed in this paper. Suppose that the polarizations of all the domains in the specimen are parallel to the $x-z$ plane. The polarizations of all the domains will not change along the $y$ direction. A unit thickness of the specimen is chosen for the $2 \mathrm{D}$ finite element calculation and uniformly distributed compressive stress is applied to the specimen on $z= \pm a / 2$. The initial polarization is nearly assigned to the positive direction of the $z$ axis. The displacements of $u_{x}$ and $u_{z}$ on point $(-a / 2,-a / 2)$ are fixed at zero. The displacement $u_{z}$ on the point $(a / 2,-a / 2)$ is fixed at zero too, as shown in Figure 1.

\subsection{The calculated model and method}

The simulation was carried out with a large number of domains. A strong electric field polarized the ceramics by aligning the polarization of the domain as closely as possible with the applied field. Each domain is represented by one element in the finite element simulation. Each element has a spontaneous polarization. The macroscopic response of the specimen is computed from the volume average response of each domain:

$$
\begin{aligned}
& \gamma_{i j}=\frac{1}{V}\left(\sum_{l=1}^{N_{\mathrm{t}}} \gamma_{i j(l)} V_{l}\right), \\
& D_{i}=\frac{1}{V}\left(\sum_{l=1}^{N_{\mathrm{t}}} D_{i(l)} V_{l}\right),
\end{aligned}
$$

where $N_{\mathrm{t}}$ is the total number of the triangle elements, $V$ is the volume of the ferroelectric body, $V_{1}$ is the volume of the $l$ th element, and $\gamma_{i j(1)}, D_{i(1)}$ is the strain and electric displacement of the $l$ th element, respectively. Since the samples of the polycrystalline ferroelectric ceramic are first poled by applying an electric field excess of the coercive field, this changes the original direction of the polarization vector of the domains to that most closely aligned with the positive direction of the $z$ axis. Hence in this paper the polarization vector of the domains is assigned nearly to the $z$ direction. Let $\varphi$ be the angle between the initial polarization vector of the domain and the $z$ axis. So the random distribution of the polarization vector orientation is achieved by randomly generating 
values for $\varphi\left(-36^{\circ} \leqslant \varphi \leqslant 36^{\circ}\right)$. Triangle elements with uniform strain and electric field are used. Each element denotes a single domain zone, i.e., the material within the element is homogeneous. The sketch of the grids is shown in Figure 2.

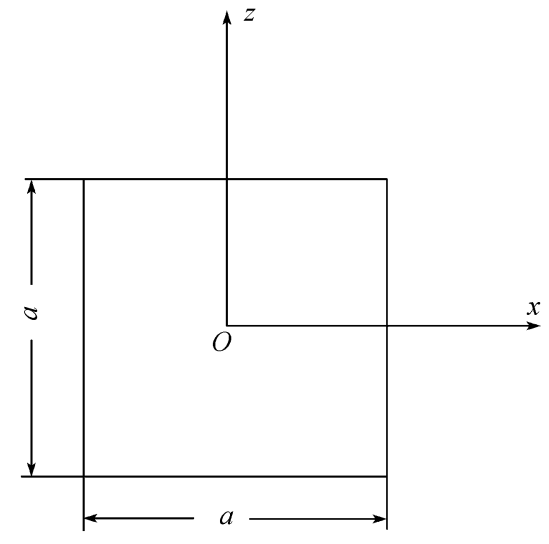

Figure 1 The sketch of the calculated model.

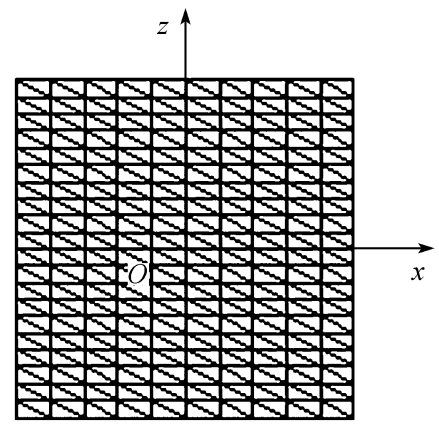

Figure 2 The sketch of finite element grids.

Polycrystalline ferroelectric ceramic (8/65/35 PLZT) was used in the experiment studied by Lynch $^{[38]}$. The material parameters measured by $\operatorname{Lynch}^{[38]}$ are $Y=68 \times 10^{9} \mathrm{~Pa}, v=0.3, \varepsilon_{33}=56.25 \times$ $10^{-9} \mathrm{C} /(\mathrm{V} \cdot \mathrm{m}), P_{r}=0.25 \mathrm{C} / \mathrm{m}^{2}, \gamma_{r}=0.143 \%, E_{c}=0.36 \mathrm{MV} / \mathrm{m}$. Hwang et al. ${ }^{[2]}$ suggested that the remnant polarization $P_{r}$ of the polycrystalline ferroelectric ceramic (8/65/35 PLZT) and the spontaneous polarization $P_{s}$ of the single ferroelectric crystal had the following empirical relationship:

$$
P_{r}=0.83 P_{s} .
$$

But in present calculation, the initial polarization of domains is distributed randomly. If the interaction of an element to its adjoining elements in the ferroelectric body is neglected, one can get the following relations:

$$
\begin{aligned}
& P_{r}=\alpha P_{s}=0.899 P_{s}, \\
& \gamma_{r}=\beta \gamma_{s}=0.726 \gamma_{s},
\end{aligned}
$$

where $\alpha=0.899$ and $\beta=0.726$ are given in Appendix A. The sketch of the distribution of the initial polarization in the whole sample is also listed in Appendix A. When the interaction between the elements is considered, in other words, the residual strain and residual electric field are taken into account, $\alpha$ and $\beta$ may change a little. We can get the residual strain and the residual electric field of each element through finite element calculation without any applied external electric field and mechanical loads.

Then we can get the remnant strain and the remnant electric displacement of the specimen. After several adjustment calculations, one can get the suitable parameters $P_{s}$ and $\gamma_{s}$ which guarantee the remnant polarization $P_{r}=0.25 \mathrm{C} / \mathrm{m}^{2}$ and $\gamma_{r}=0.143 \%$. Then we have

$$
\begin{array}{cc}
P_{s}=0.302 \mathrm{C} / \mathrm{m}^{2}, & P_{r}=0.828 P_{s}, \\
\gamma_{s}=0.203 \%, & \gamma_{r}=0.704 \gamma_{s} .
\end{array}
$$


The parameters $\alpha=0.828$ and $\beta=0.704$ which are close to the values given by Hwang et al. ${ }^{[6]}$. Hence in this paper for the ferroelectric single domain (PLZT 8/65/35), the material parameters are chosen to be $Y=60 \times 10^{9} \mathrm{~Pa}, v=0.3, \varepsilon_{33}=62 \times 10^{-9} \mathrm{C} /(\mathrm{V} \cdot \mathrm{m}), P_{s}=0.302 \mathrm{C} / \mathrm{m}^{2}, \gamma_{s}=0.203 \%$, $E_{c}=0.36 \mathrm{MV} / \mathrm{m}, d_{33}=0.9 * 10^{-9} \mathrm{~m} / \mathrm{V}, d_{31}=-4.5 \times 10^{-10} \mathrm{~m} / \mathrm{V}, d_{15}=1.545 \times 10^{-9} \mathrm{~m} / \mathrm{V}$.

The nonlinear constitutive behavior of the ferroelectric specimen is calculated by two methods. First, no interaction between the adjacent domains in the specimen is taken into account. The stress and electric field for each element in the specimen are assumed to be equal to the applied stress and electric field. Then the driving force of domain switching and the hardening function are calculated. The volume fraction of domain switching is calculated by using formulas (8)-(12). The strain and electric displacement of each element is gained by substituting the electric field and stress and the volume fraction of domain switching into constitutive eqs. (1) and (3). The macroscopic strain and electric displacement of specimen is obtained by volume averaging. In the second method, the driving force of domain switching is also calculated by using the applied electric field and stress. The volume fraction of domain switching is calculated by using formulas (8)-(12). Based on this volume fraction of domain switching, one can carry out the finite element calculation for the ferroelectric specimen using the variational principle and get the strain $\gamma_{i j}$ and electric field $E_{i}$ for

all elements. The contribution of each element to the macroscopic behavior of the specimen is then calculated by volume averaging.

\section{The calculated results}

The nonlinear constitutive behavior of the ferroelectric body subjected to the axial electromechanical loading is studied in this section. The comparison of simulated and measured results is shown in Figures 3-12. The solid lines in Figures 3-12 correspond to the experimental results of Lynch $^{[38]}$ and the present calculation results given by the first method are plotted as the open circles. The FEM results are shown by the open triangles.

\subsection{Uniaxial mechanical field loading}

We divide the load $-85 \mathrm{MPa}$ into 100 steps. The external mechanical loading is increasing step by step. Figures 3 and 4 show that the behaviors of the stress versus electric displacement and the

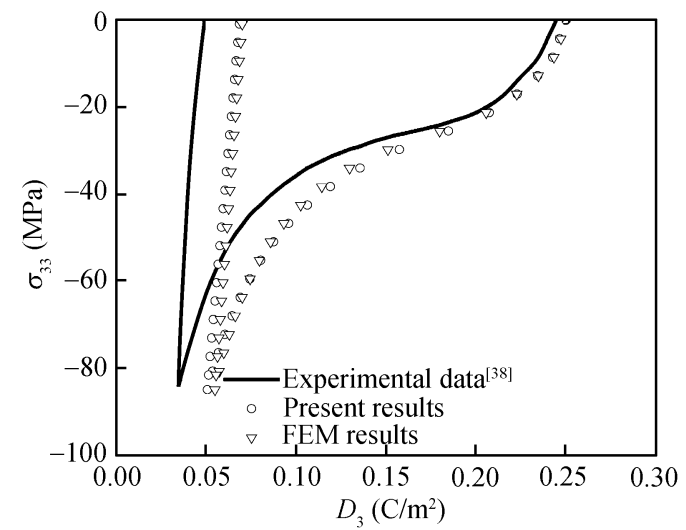

Figure 3 Simulated and measured stress vs. electric displacement at zero applied electric field.

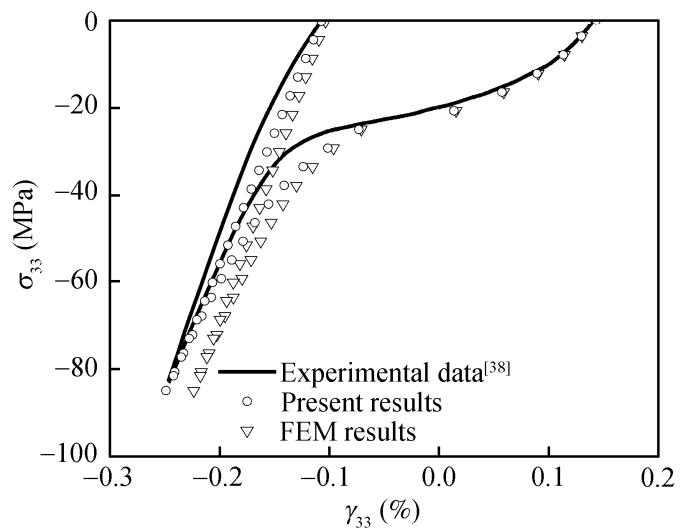

Figure 4 Simulated and measured stress vs. strain at zero applied electric field. 
stress versus strain are linear when the applied compressive stress is less than the critical value $\sigma_{c}$. In our simulation, there is no domain switching before the applied compressive stress less than the critical value $\sigma_{c}$. The initial slope of the stress-electric displacement curve is the piezoelectric coefficient $d_{33}$. The initial slope of the stress-strain curve is the Young's modulus. The $90^{\circ}$ switching takes place when the uniaxial compressive stress exceeds the critical value $\sigma_{c}$.

Now we discuss the unloading process from $\sigma_{33}=-85 \mathrm{MPa}$. In our simulation no domain switching occurs during the unloading. Hence the behavior of the stress-electric displacement and the stress-strain becomes linear again. The slope of the stress-electric displacement curve is close to infinity ( $d_{33}$ is nearly zero) and the slope of the stress-strain curve is Young's modulus again during the unloading. Figure 3 shows that the calculated electric displacement versus the applied compressive stress curve for the specimen is consistent with the experimental results for the case $\left|\sigma_{33}\right|<30 \mathrm{MPa}$. When $\sigma_{33}$ is less than $-30 \mathrm{MPa}$, the calculated results begin to deviate from the experimental curve. At $-85 \mathrm{MPa}$ the calculated electric displacement $D_{3}$ is less than the experimental result. Figure 4 shows the axial stress-strain curve. When $\left|\sigma_{33}\right|$ is less than $30 \mathrm{MPa}$, the calculated results in two methods are in good agreement with the experimental results. With the increase of the compressive stress, the calculated axial strain $\gamma_{33}$ of FEM results deviates from the experimental curve gradually. The reason is described as follows. The angle $\varphi$ between the direction of polarization and the $z$ axis is 0 in the single domain mechanical model. But the orientation of domains is achieved by randomly generating the values for $\varphi$ between $-\pi / 5$ and $\pi / 5$ in the multi-domain model. Hence in the same loading, the driving force in the single domain mechanical model is greater than those in the multi-domain mechanical model. In the same way, the volume fraction of domain switching in the single domain mechanical model is greater than those in the multi-domain mechanical model.

\subsection{Uniaxial electric field loading}

Figures 5 and 6 show the ferroelectric hysteresis and the butterfly shaped curve under the uniaxial electric loading, respectively. First we discuss the negative electric field loading ( $E_{3}$ opposite to the direction of the remnant polarization of the specimen). From Figure 5 we can see that the calculated axial strain $\gamma_{33}$ agrees well with the experimental results from point $A$ to points $B$ and $C$. As shown in Figure 6, the calculated electric displacement $D_{3}$ is in good agreement with the experimental results before $E_{c}$. Now a positive electric field is applied to the specimen from point $D$. With the increase of electric field $E_{3}$, the domain switching occurs in some elements. The situation of domain switching is similar to that of the negative electric loading from point $A$ to points $B$ and $C$. The computed strain $\gamma_{33}$ and electric displacement $D_{3}$ for the specimen agree well with the experimental data from point $E$ to point $F$. The computed strain $\gamma_{33}$ and electric displacement $D_{3}$ for the specimen match well with the experimental data from point $F$ and point $A$.

\subsection{Combined electromechanical loading}

As for the electromechanical coupling loading, a prescribed compressive stress is applied to the specimen first. Then a circle of electric field is applied to the specimen, while the external com- 


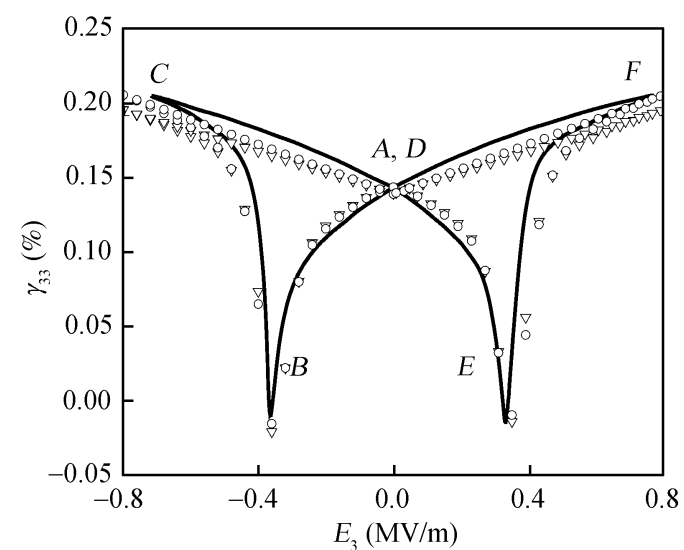

Figure 5 Comparison of simulated and measured strain vs. electric field curves at $\sigma_{33}=0$.

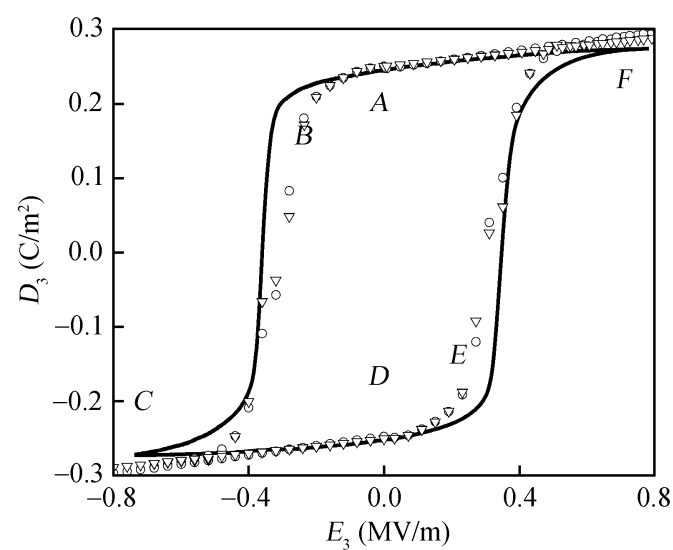

Figure 6 Comparison of simulated and measured electric displacement vs. electric field curves at $\sigma_{33}=0$.

pressive stress is held fixed. Figures 7-12 show the results of the experimental and the calculated electric displacement and the strain versus the applied electric field with different compressive stress loadings. The solid lines in Figures 7-12 correspond to the experimental results obtained by Lynch $^{[38]}$ and the present calculation results are plotted as the open circles. The FEM results are shown by the open triangles.

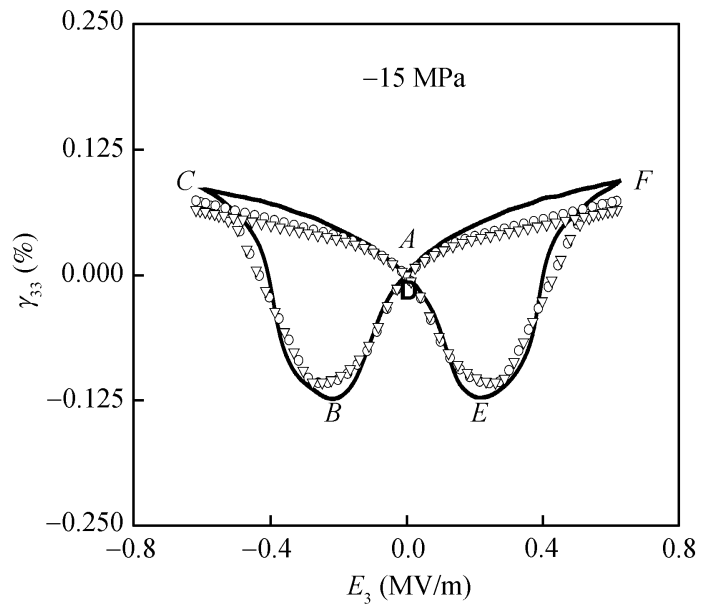

Figure 7 Comparison of simulated and measured strain vs. electric field curves at $\sigma_{33}=-15 \mathrm{MPa}$.

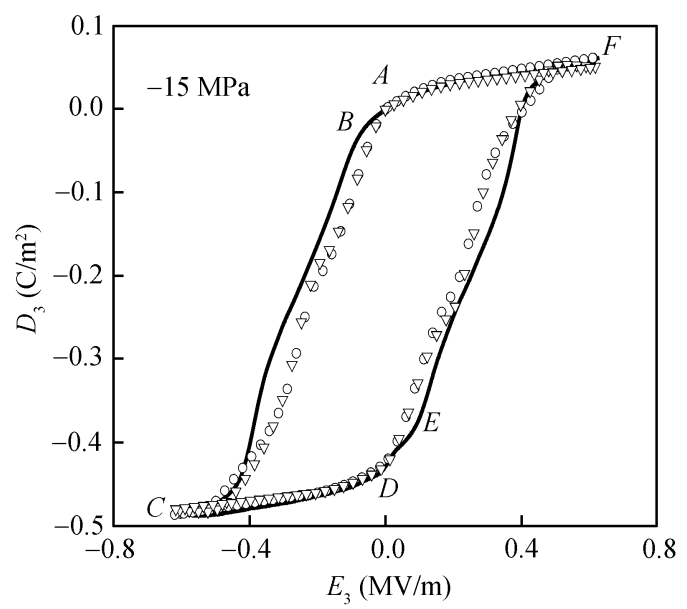

Figure 8 Comparison of simulated and measured electric displacement vs. electric field curves at $\sigma_{33}=-15 \mathrm{MPa}$.

First we discuss the case of $\sigma_{33}=-15 \mathrm{MPa}$. This prescribed compressive stress is applied to the specimen and held fixed. An electric field parallel to the applied stress is then introduced and cycled between the positive and the negative limits. The general feature of the hysteresis loop and the butterfly loop for a constant stress of $-15 \mathrm{MPa}$ is captured quite well in the predicted curves.

For the cases of $\sigma_{33}=-30 \mathrm{MPa}$ and $-60 \mathrm{MPa}$, the comparison of the simulated and the measured longitudinal strain versus the electric field is satisfying. But the remnant polarization is predicted relatively poor in the calculated curves. This inaccuracy is probably associated with the deficiency of the model in handling $180^{\circ}$ switching. A simple linear fit of the hardening relation of $180^{\circ}$ switching cannot reflect the actual evolution process of the domain well. 


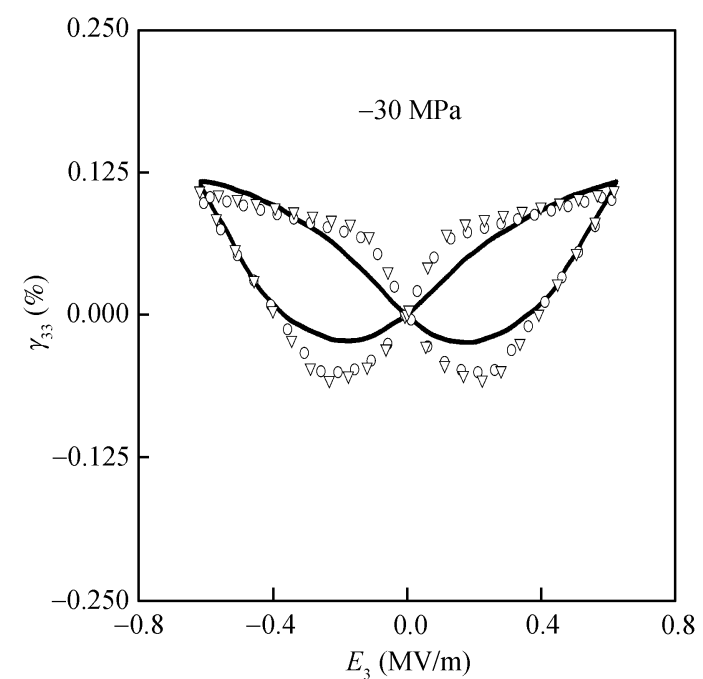

Figure 9 Comparison of the simulated and the measured strain versus the electric field curves at $\sigma_{33}=-30 \mathrm{MPa}$.

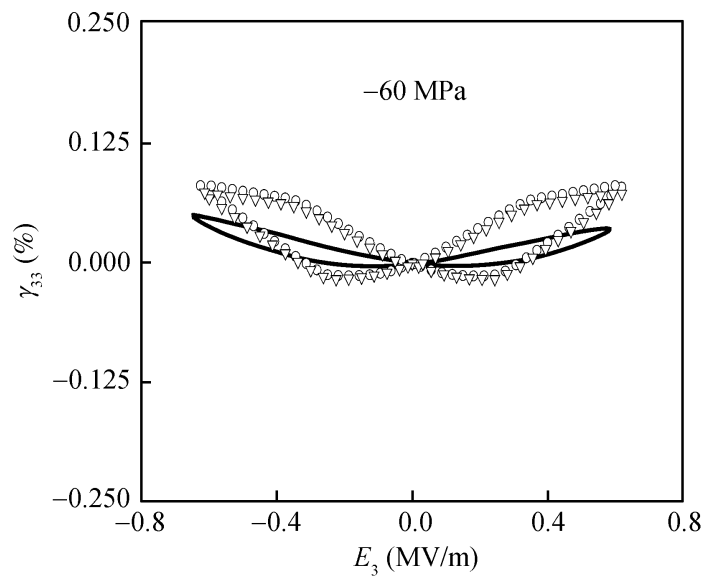

Figure 11 Comparison of the simulated and the measured strain versus the electric field curves at $\sigma_{33}=-60 \mathrm{MPa}$.

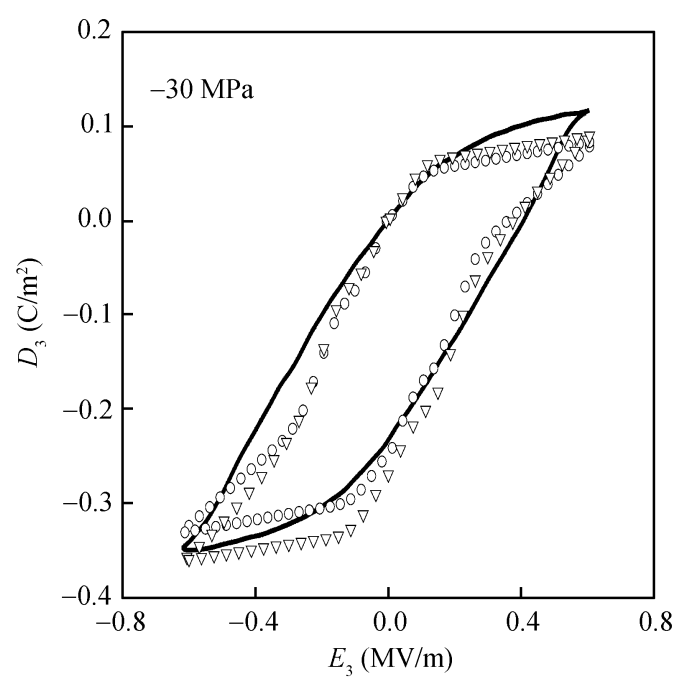

Figure 10 Comparison of the simulated and the measured electric displacement versus the electric field curves at $\sigma_{33}=$ $-30 \mathrm{MPa}$.

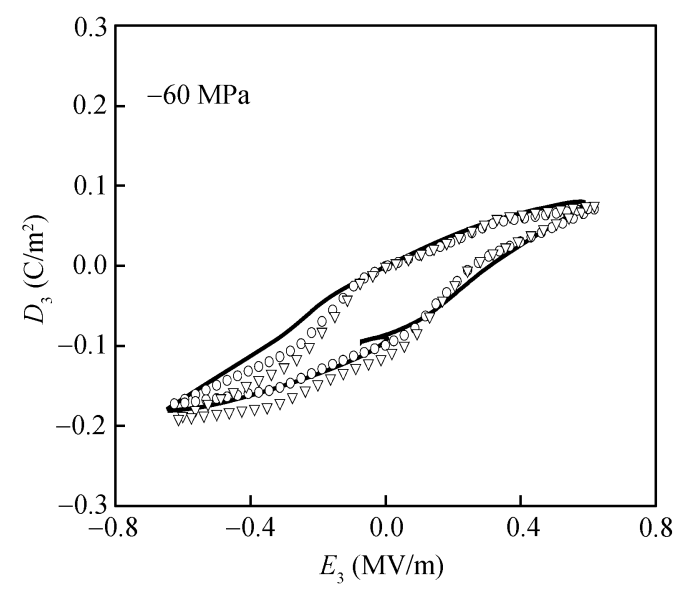

Figure 12 Comparison of the simulated and the measured electric displacement versus the electric field curves at $\sigma_{33}$ $=-60 \mathrm{MPa}$.

\section{Conclusions}

The multi-domain mechanical model is developed in this work. By using the hardening relation between the driving force of domain switching and the volume fraction of domain switching calibrated by ref. [1], the nonlinear response of the ferroelectric specimen is calculated in two methods. First, based on the multi-domain mechanical model, each domain is represented by one element. No interaction of a domain with its adjacent domain in the specimen is taken into account, thus each element is free of any constraint. The driving force of domain switching and the hardening function are calculated. The volume fraction of domain switching is calculated by using formulas (8)-(12). The strain and electric displacement of each element are gained by substituting electric field and stress and the volume fraction of domain switching into the constitutive equation. The 
The volume average strain and electric displacement are calculated. In the second method, the driving force of domain switching is also calculated by using the applied electric field and/or stress. The volume fraction of domain switching is also calculated by using formulas (8)-(12). Based on this value of volume fraction of domain switching, one can carry out the finite element calculation using the variational principle and get the strain $\gamma_{i j}$ and electric field $E_{i}$. After each increment of the applied field and updating the polarization, the macroscopic response of the ceramic is computed from the volume average response of each element. A simplified 2D finite element model is presented in this paper and compared to the experimental results by Lynch $^{[38]}$. A systematic finite element analysis is performed on the nonlinear behavior of the ferroelectric ceramics subjected to the electromechanical coupling loading. The results involve the electric butterfly shaped curves of the axial strain versus the axial electric field, the hysteresis loops of the electric displacement versus the electric field and the evolution process of domain switching in the ferroelectric specimen under the uniaxial coupled stress and electric field. The present theoretic prediction reasonably agrees with the experimental results given by $\operatorname{Lynch}^{[38]}$.

\section{Appendix A}

The coefficients $\alpha, \beta$ are given by

$$
\begin{gathered}
\alpha=\sum_{i=1}^{N} \cos \varphi_{i} / N, \\
\beta=\sum_{i=1}^{N}\left(\cos ^{2} \varphi_{i}-\frac{1}{2} \sin ^{2} \varphi_{i}\right) / N,
\end{gathered}
$$

where $\varphi_{i}$ is the angle between the initial polarization vector of $i$ th domain and the $z$ axis, and $N$ is the total number of the triangle elements. Random orientation of the elements is achieved by randomly generating values for $\varphi_{i}$ between $-\pi / 5$ and $\pi / 5$. Figure 13 is a schematic diagram for the orientation of $\varphi_{i}$, the horizontal coordinate is the number $k$, and the longitudinal coordinate is the number $N_{k}$ of the elements whose orientation angle satisfies $(k-3)^{\circ} \leqslant \varphi_{i}<k^{\circ}(k=-33,-30,-27, \ldots$, $33,36)$.

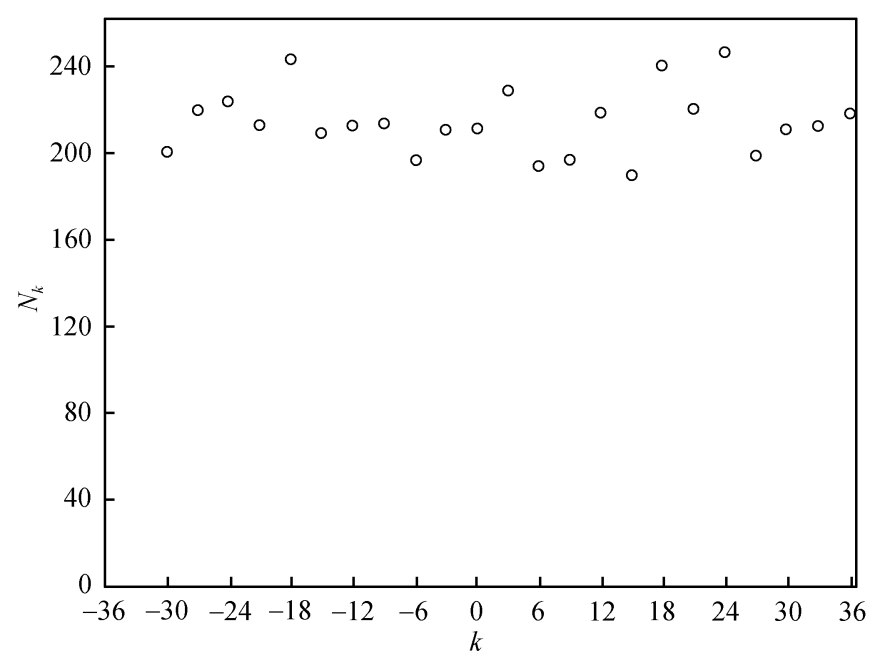

Figure 13 Schematic diagram for the orientation of $\varphi_{i}$. 
1 Liu F, Li H J, Wang T C. Energy principle of ferroelectric materials and single domain mechanical model. Acta Mech Sin, 2007, 23: 531-543[DOI]

2 Hwang S C, Lynch C S, McMeeking R M. Ferroelectric/ferroelastic interactions and a polarization switching model. Acta Metall Mater, 1995, 43: 2073-2084[DOI]

3 Chan K, Hagood N. Modeling of nonlinear piezoceramics for structural actuation. Proc SPIE Svmp Smart Struct Mater, 1994, 2190: 194-205

4 Lu W, Fang D N, Hwang K C. Nonlinear electric-mechanical behavior and micormechanics modeling of ferroelectric domain evolution. Acta Mater, 1999, 47: 2913-2926[DOI]

5 Steinkopff T. Finite-element modeling of ferroelectric domain switching in piezoelectric ceramics. J Euro Ceram Soc, 1999, 19: 1247-1249[DOI]

6 Hwang S C, Huber J E, McMeeking R M, et al. The simulation of switching in polycrystalline ferroelectric ceramics. J Appl Phys, 1998, 84: 1530-1540[DOI]

7 Chen X, Fang D N, Hwang K C. Micromechanics simulation of ferroelectric polarization switching. Acta Mater, 1997, 45: 3181-3189[DOI]

8 Huo Y Z, Jiang Q. Modeling of domain switching in ferroelectric ceramics: An example. Int J Solids Struct, 1998, 35: $1339[\mathrm{DOI}]$

9 Zhang Z K, Fang D N, Soh A K. A new criterion for domain-switching inferroelectric materials. Mech Mater, 2006, 38: $25-32[\mathrm{DOI}]$

10 Shaikh M G, Phanish S, Sivakumar S M. Domain switching criteria for ferroelectrics. Comput Mater Sci, 2006, 37: 178$186[\mathrm{DOI}]$

11 Huber J E, Fleck N A, Landis C M, et al. A constitutive model for ferroelectric polycrystals. J Mech Phys Solids, 1999, 47: $1663-1697[\mathrm{DOI}]$

12 Huber J E, Fleck N A. Multi-axial electrical switching of a ferroelectric: Theory versus experiment. J Mech Phys Solids, 2001, 49: 785-811[DOI]

13 Chen X, Fang D N, Hwang K C. Micromechanics simulation of ferroelectric polarization switching. Acta Mater, 1997, 45: $3181-3189[\mathrm{DOI}]$

14 Lu W, Fang D N, Hwang K C. Micromechanics of ferroelectricdomain switching behavior. Part I: Coupled electromechanical field of domain inclusions. Theor Appl Fract Mech, 2001, 37: 29-38[DOI]

15 Lu W, Fang D N, Hwang K C. Micromechanics of ferroelectricdomain switching behavior. Part II: Constitutive relations and hysteresis. Theor Appl Fract Mech, 2001, 37: 39-47[DOI]

16 Chen P J, Peercy P S. One dimensional dynamic electromechanical constitutive relations of ferroelectric materials. Acta Mech, 1979, 31(4): 231-241[DOI]

17 Chen P J, Madsen M M. One dimensional polar response of the electrooptic PLZT 7/65/35 due to domain switching. Acta Mech, 1981, 41: 255-264[DOI]

18 Chen P J, Mongomery S T. A macroscopic theory for the existence of the hysteresis and butterfly loops in ferroelectricity. Ferroelectrics, 1980, 23: 199-208

19 Chen P J, Tucker T J. Determination of the polar equilibrium properties of the ferroelectric ceramic PZT 65/35. Acta Mech, 1981, 38: 209-218[DOI]

20 Bassiouny A F, Ghaleb G, Maugin G. Thermodynamical formulation for coupled electromechanical hysteresis effects (I): Basic equations. Int J Engng Sci, 1988, 26(12): 1279-1295[DOI]

21 Bassiouny A F, Ghaleb G, Maugin G. Thermodynamical formulation for coupled electromechanical hysteresis effects (II): Poling of ceramics. Int J Engng Sci, 1988, 26(12): 1297-1306[DOI]

22 Kamlah M, Tsakmakis C. Phenomenological modeling of the non-linear electromechanical coupling in ferroelectrics. Int J Solids Struct, 1999, 36(5): 669-695[DOI]

23 Kamlah M, Böhle U, Munz D. On a non-linear finite element method for piezoelectric structures made of hysteretic ferroelectric ceramics. Comput Mater Sci, 2000, 19: 81-86[DOI]

24 Kamlah M, Böhle U. Finite element analysis of piezoceramics components taking into account ferroelectric hysteresis behavior. Int J Solids Struct, 2001, 38(4): 605-633[DOI]

25 Kamlah M, Wang Z G. A thermodynamically and microscopically motivated constitutive model for piezoceramics. Comput Mater Sci, 2003, 28: 409-418[DOI] 
26 Landis C M, McMeeking R M. A phenomenological constitutive law for ferroelastic switching and a resulting asymptotic Crack Tip Solution. J Intel Mater Sys Struct, 1999, 10: 155-163[DOI]

27 Cocks A C F, McMeeking R M. A phenomenological constitutive law for the behavior of ferroelectric ceramics. Ferroelectrics, 1999, 228: 219-228[DOI]

28 McMeeking R M, Landis C M. A phenomenological multi-axial constitutive law for switching in polycrystalline ferroelectric ceramics. Int J Engng Sci, 2002, 40: 1553-1577[DOI]

29 Landis C M. Fully coupled, multi-axial, symmetric constitutive laws for polycrystalline ferroelectric ceramics. J Mech Phys Solids, 2002, 50: 127-152[DOI]

30 Kamlah M. Ferroelectric and ferroelastic piezoceramics-modeling of electromechanical hysteresis phenomena. Continuum Mech Thermodyn, 2001, 13(4): 219-268

31 Landis C M. Non-linear constitutive modeling of ferroelectrics. Curr Opin Solid State Mater Sci, 2004, 8: 59-69[DOI]

32 Gong X, Suo Z. Reliability of ceramic multilayer actuators: a nonlinear finite element simulation. J Mech Phys Solids, 1996, 44(5): 751-769[DOI]

33 Hom C L, Shankar N. A finite element method for electrostrictive ceramic devices. Int J Solids Struct, 1996, 33, 17571779 [DOI]

34 Hwang S C, Waser R. Study of electrical and mechanical contribution to switching in ferroelectric/ferroelastic polycrystals. Acta Mater, 2000, 48(12): 3271-3282[DOI]

35 Liu B. Study on the fracture and fatigue of ferroelectric materials (in Chinese). Dissertation for the Doctoral Degree. Beijing: Tsinghua University, 2000. 82-97

36 Li F X, Fang D N. Simulations of domain switching in ferroelectrics by a three-dimensional finite element model. Mech Mater, 2004, 36(10): 959-973[DOI]

37 Cao H, Evans A G. Nonlinear deformation of ferroelectric ceramics. J Am Ceram Soc, 1993, 76(4): 890-896 [DOI]

38 Lynch C S. The effect of uniaxial stress on the electro-mechanical response of 8/65/35 PLZT. Acta Mater, 1996, 44(10): 4137-4148[DOI]

39 Fang D N, Li C Q. Nonlinear electric-mechanical behavior of a soft PZT-51 ferroelectric ceramic. J Mater Sci, 1999, 34(4): 4001-4010[DOI]

40 Miller R C, Weinreich G. Mechanism for the sidewise motion of $180^{\circ}$ domain walls in barium titanate. Phys Rev, 1960, 117: $1460-1466[\mathrm{DOI}]$

41 Hayashi M. Kinetics of domain wall motion in ferroelectric switching (I): General formulation. J Phys Soc Jpn, 1972, 33: $616-628[\mathrm{DOI}]$

42 Zhong W L. Physics of Ferroelectrics (in Chinese). Beijing: Science Press, 1996 